\title{
HOMOTOPY THEORY OF CYCLIC PRESHEAVES
}

\author{
SIGURD SETEKLEV and PAUL ARNE ØSTVÆR
}

\begin{abstract}
We generalize the homotopy theory of cyclic sets to cyclic presheaves on small Grothendieck sites. This is achieved by constructing pointwise and local model structures reminiscent of the homotopy theory of simplicial presheaves.
\end{abstract}

\section{Introduction}

The works of Dwyer, Hopkins and Kan [7] and Spaliński [14] show there are Quillen equivalences between model structures on topological spaces with a circle action and cyclic sets. In this paper we extend their work in an arithmetic direction by constructing model structures on cyclic presheaves on small Grothendieck sites [12]. Connes' cyclic homology, Bökstedt's topological Hochschild homology, Goodwillie's cyclic $K$-theory and the computational successes of Jardine's homotopy theory of simplicial presheaves [11] provide much motivation and point towards applications of these model structures.

In our construction of model structures on cyclic presheaves the definitions are rigged such that fibrant objects admit characterizations similar to fibrant simplicial presheaves. Such characterizations are of interest because descent questions, e.g. in the simplicial case the Quillen-Lichtenbaum conjecture for algebraic and étale $K$-theory deal with fibrancy conditions. By way of example, suppose $\left\{U^{\alpha} \rightarrow X\right\}$ is an open cover of a scheme $X$. Then algebraic $K$-theory $K$ satisfies Zariski descent because the functorially induced map between $K(X)$ and the homotopy limit of the simplicial Čech complex of the cover is a weak equivalence:

$$
K(X) \stackrel{\sim}{\longrightarrow} \operatorname{holim}\left(\prod K\left(\mathscr{U}^{\alpha}\right) \Longrightarrow \prod K\left(\mathscr{U}^{\alpha} \cap \mathscr{U}^{\alpha^{\prime}}\right) \rightrightarrows \cdots\right)
$$

This approach necessitates many formal similarities with the homotopy theory of simplicial presheaves presented in [5] and [10]. The first section recalls and expands the works on homotopy theory of cyclic sets. Second, we introduce cyclic presheaves on small Grothendieck sites and work out the model 
structures. Cyclic presheaves are simplicial presheaves equipped with a cyclic operator subject to the usual cyclic identities. The latter leads naturally to the notion of a cyclic model structure, which is derived from the well known notion of a simplicial model structure. As a consequence of the existence of the model structures, every cyclic presheaf is weakly equivalent to some cyclic presheaf that satisfies a certain descent condition reminiscent of the $K$-theoretic example of Zariski descent. A discussion of descent for cyclic $K$-theory seems to be missing in the literature. We note that cyclic sets are cyclic presheaves on the one-point site, but the more general setup allows to include everyday algebro-geometric examples arising from Grothendieck topologies.

A more detailed presentation of the content of this paper can be found in the first author's master's thesis [13]. He was partially supported by an Abel stipend granted by the Norwegian Mathematical Society. The authors gratefully acknowledge the excellent working conditions and support provided by the Fields Institute during the spring 2007 Thematic Program on Geometric Applications of Homotopy Theory. We wish to thank Clark Barwick for sharing his many insights on model categories, in particular [2], Bjørn Ian Dundas for helpful emendations on [13] and Jan Spaliński for explaining his work on cyclic sets [14].

\section{Cyclic sets}

Let $\Lambda$ denote Connes' cyclic category containing the category $\Delta$ of finite ordinal numbers and all finite cyclic groups [4]. Denote by $\Lambda$ Set the functor category of cyclic sets or contravariant functors from $\Lambda$ to the category Set of sets. If $n \geq 0$, let $\Lambda[n]$ denote the standard cyclic set $\Lambda^{\mathrm{op}}(\mathbf{n},-): \Lambda^{\mathrm{op}} \rightarrow$ Set. The Yoneda lemma implies there are natural isomorphisms $\Lambda \operatorname{Set}(\Lambda[n], K) \cong$ $K_{n}$ for every cyclic set $K$. The inclusion $i: \Delta^{\mathrm{op}} \subset \Lambda^{\mathrm{op}}$ induces an adjunction where the left adjoint $i$ ! is a left Kan extension:

$$
i_{!}: \Delta \text { Set } \rightleftarrows \Lambda \text { Set }: i^{*}
$$

By [7], defining weak equivalences and fibrations between cyclic sets via the right adjoint forgetful functor $i^{*}$ yields the so-called "weak" model structure on $\Lambda$ Set. The weak cofibrations are generated by the set of maps $i_{!}(\partial \Delta[n] \subset$ $\Delta[n])$ for $n \geq 0$ and the acyclic weak cofibrations by the set of maps $i_{!}\left(\Delta^{k}[n] \subset\right.$ $\Delta[n])$ for $n \geq 1,0 \leq k \leq n$. Thus the weak model structure is combinatorial, cf. [2] and [3]. The cofibrations are special types of monomorphisms that admit a combinatorial description detailed in [7, Proposition 3.5].

For every integer $r \geq 1$ there exists an adjunction noted in [14, §3]:

$$
\Psi_{r}: \Delta \text { Set } \rightleftarrows \Lambda \text { Set: } \Phi_{r}
$$


For a cyclic set $K$ we have $\Phi_{r}(K)=\left(\operatorname{sd}_{r} K\right)^{C_{r}}$ where $\operatorname{sd}_{r}(K)_{n} \equiv K_{r(n+1)-1}$ is the $r$-fold subdivision of the underlying simplicial set equipped with the natural action of the cyclic group $C_{r}$ of order $r$ [14, §3.7]. The geometric realization of $\Phi_{r}(K)$ is homeomorphic to the fixed points of $C_{r}$ acting on the topological realization of $K$. We note $\Psi_{r}$ preserves monomorphisms because it maps the generating cofibrations $\partial \Delta[n] \subset \Delta[n]$ to monomorphisms: Applying $\Psi_{r}$ to some face map $\Delta[n-1] \rightarrow \Delta[n]$ yields a monomorphism by comparison with a degeneracy map. Note that $\partial \Delta[n]$ is the direct limit of a diagram comprising copies of $\Delta[n-2]$ and $\Delta[n-1]$ together with face maps. If we apply $\Psi_{r}$ to this diagram, the maps in the resulting diagram will be monomorphisms. Since $\Psi_{r} \Delta[n-1] \rightarrow \Psi_{r} \Delta[n]$ is a monomorphism, so is the induced map $\Psi_{r} \partial \Delta[n] \rightarrow \Psi_{r} \Delta[n]$ on the colimit. Spaliński has shown that the functors $\Phi_{r}$ for every $r \geq 1$ collectively define a combinatorial model category structure on $\Lambda$ Set by comparison with simplicial sets [14, Theorem 3.10]. It is called the "strong" model structure on cyclic sets since it is Quillen equivalent to the fine model structure on $S^{1}$-spaces where weak equivalences and fibrations are detected on fixed points of strict closed subgroups of $S^{1}$ [14, Theorem 5.1]. The proof shows that defining weak equivalences and fibrations on cyclic sets using $\Phi_{r}$ for some fixed integer $r \geq 1$ yields a "weak level $r$ " combinatorial model structure with generating cofibrations $\Psi_{r}(\partial \Delta[n] \subset \Delta[n])$ for $n \geq 0$, and generating acyclic cofibrations $\Psi_{r}\left(\Delta^{k}[n] \subset \Delta[n]\right)$ for $n \geq 1,0 \leq k \leq n$, induced up from simplicial sets. More precisely, a map between cyclic sets $K \rightarrow L$ is declared to be a level $r$ weak equivalence if the map $\Phi_{r}(K \rightarrow L)$ is a weak equivalence between simplicial sets, and likewise for the fibrations.

The classes of weak equivalences in the weak and strong model structures form full accessible subcategories of the morphism category $\operatorname{Mor}(\Lambda$ Set $)$, cf. [1], [2], [3]. Moreover, there exists a set of monomorphisms $I$ in $\Lambda$ Set such that $\operatorname{cof}(I)$ - that is, maps with the left lifting property with respect to every map having the right lifting property with respect to every member in $I[9$, Definition 2.1.7] - coincides with the class of monomorphisms in $\Lambda$ Set [3, Proposition 1.12]. Since the cofibrations in the weak level $r$ and strong model structures on $\Lambda$ Set are monomorphisms, we may arrange that $I$ contains the set of generating cofibrations obtained by applying $\Psi_{r}$ to the generating cofibrations in $\Delta$ Set for $r$ respectively every $r \geq 1$. It follows that the class of maps $\operatorname{inj}(I)-$ defined by the right lifting property with respect to $I[9$, Definition 2.1.7] - consists of weak equivalences.

Combining the above with Jeff Smith's main theorem on combinatorial model categories [3, Theorem 1.7, Proposition 1.15] we conclude the following result holds: 
THEOREM 2.1. The following classes of weak equivalences together with the monomorphisms as cofibrations define "weak monic level $r$ " and "strong monic" combinatorial and proper model structures on cyclic sets.

(i) The level $r$ weak equivalences.

(ii) The maps that are level $r$ weak equivalences for every $r \geq 1$.

The identity functor on $\Lambda$ Set yields Quillen equivalences between the weak and weak monic model structures and between the strong and strong monic model structures. The adjunction (2) is a Quillen pair for the weak monic level $r$ model structure.

Right properness of the model structures in Theorem 2.1 follows using (2) and right properness of simplicial sets, while left properness holds since every object is cofibrant. In fact, Spaliński's model structures on cyclic sets are also proper: Left properness holds since $\Phi_{r}$ preserves pushouts of diagrams of the form $L \leftarrow K \subseteq K^{\prime}$ and the model structure on simplicial sets is left proper. Likewise, the next result holds true because the model structure on simplicial sets is monoidal. A "monic model structure" refers to any of the model structures on $\Lambda$ Set established in Theorem 2.1.

LEMMA 2.2. The monic model structures on $\Lambda$ Set are monoidal: Ifi: $K \rightarrow L$ and $i^{\prime}: K^{\prime} \rightarrow L^{\prime}$ are cofibrations, then the pushout product map

$$
L \times K^{\prime} \coprod_{K \times K^{\prime}} K \times L^{\prime} \rightarrow L \times L^{\prime}
$$

is a cofibration that is a weak equivalence if in addition either $i$ or $i^{\prime}$ is so.

Observe that $\Lambda$ Set is a closed symmetric monoidal category with respect to the cartesian product when the internal hom object $\underline{\operatorname{Hom}}_{\Lambda \text { Set }}(K, L)$ of cyclic sets $K$ and $L$ is defined by $\underline{\operatorname{Hom}}_{\Lambda \text { Set }}(K, L)_{n} \equiv \Lambda \operatorname{Set}(K \times \Lambda[n], L)$; its cyclic structure is induced from the standard cyclic sets.

A cyclic model category is defined similarly to a simplicial model category by an evident "cyclic version" of Quillen's SM7 axiom. Thus a standard adjunction argument combined with Lemma 2.2 implies:

Corollary 2.3. The model structures on $\Lambda$ Set are cyclic: If $i: K \rightarrow L$ is a cofibration and $i^{\prime}: K^{\prime} \rightarrow L^{\prime}$ a fibration, then the pullback map

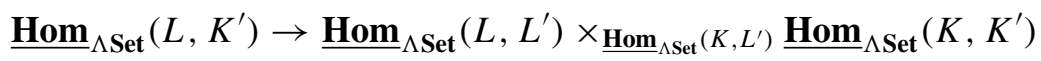

is a fibration that is a weak equivalence if in addition either $i$ or $i^{\prime}$ is so.

Following [6] let $R H(\partial \Delta[n], \Delta[n])$ denote the pushout in $\Delta$ Set of the diagram:

$$
\partial \Delta[n] \leftarrow \partial \Delta[n] \times \Delta[1] \rightarrow \Delta[n] \times \Delta[1]
$$


Using [6, Proposition 4.1] and the adjunction (2) we deduce a basepoint free lifting criterion for level $r$ weak equivalences between cyclic sets.

Lemma 2.4. Suppose $K$ and $L$ are cyclic sets such that $\Phi_{r} K$ and $\Phi_{r} L$ are Kan complexes. Then $K \rightarrow L$ is a level $r$ weak equivalence if and only if there exist liftings in all diagrams of the form:

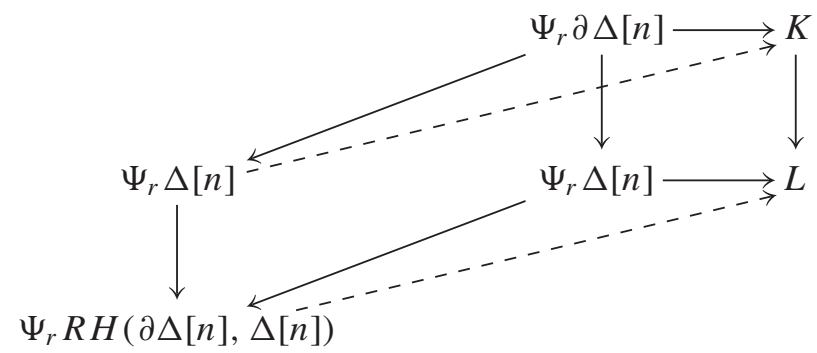

This finishes the precursors on cyclic sets and we are now ready to discuss the more general setting of cyclic presheaves.

\section{Cyclic presheaves}

Let $\mathscr{C}$ be a small Grothendieck site. The standard algebro-geometric examples are the big and small Zariski, Nisnevich, étale and h- sites of some scheme. A cyclic presheaf on $\mathscr{C}$ is a contravariant functor from $\mathscr{C}$ to $\Lambda$ Set. Note that every cyclic set defines a constant cyclic presheaf and every object of $\mathscr{C}$ represents a discrete cyclic presheaf. Denote by $\Lambda$ Pre $(\mathscr{C})$ the functor category of cyclic presheaves on $\mathscr{C}$.

Note that $\Lambda \operatorname{Pre}(\mathscr{C})$ is tensored, cotensored and enriched in cyclic sets: If $\mathscr{X}, \mathscr{Y}$ are cyclic presheaves and $K$ a cyclic set, define $\mathscr{X} \otimes K$ by $\mathscr{X} \otimes K(C) \equiv$ $\mathscr{X}(C) \times K$ and $\mathscr{Y}^{K}$ by $\mathscr{Y}^{K}(C) \equiv \underline{\mathbf{H o m}}_{\Lambda \mathrm{Set}}(K, \mathscr{Y}(C))$. Reminiscent of the internal hom objects in $\Lambda$ Set, the function complex $\operatorname{hom}_{\Lambda \text { Set }}(\mathscr{X}, \mathscr{Y})$ is defined in degree $n$ by setting $\operatorname{hom}_{\Lambda \text { Set }}(\mathscr{X}, \mathscr{Y})_{n} \equiv \Lambda \operatorname{Pre}(\mathscr{C})(\mathscr{X} \times \Lambda[n], \mathscr{Y})$. The contravariant functor on $\Lambda \operatorname{Pre}(\mathscr{C})$ given by $\mathscr{Z} \mapsto \Lambda \operatorname{Pre}(\mathscr{C})(\mathscr{X} \times \mathscr{Z}, \mathscr{Y})$ is representable by the internal hom object $\underline{\operatorname{Hom}}_{\Lambda \mathbf{P r e}(\mathscr{C})}(\mathscr{X}, \mathscr{Y})$ from $\mathscr{X}$ to $\mathscr{Y}$. With these definitions there is a natural isomorphism

$$
\underline{\operatorname{Hom}}_{\Lambda \operatorname{Pre}(\mathscr{C})}(\mathscr{X}, \mathscr{Y})(C) \cong \operatorname{hom}_{\Lambda \operatorname{Set}}(\mathscr{X}, \mathscr{Y}(C \times-))
$$

for every object $C$ of $\mathscr{C}$.

The cyclic and simplicial categories of presheaves on $\mathscr{C}$ are also related by adjoint functors for every integer $r \geq 1$ :

$$
\Psi_{r}^{\mathscr{C}}: \Delta \operatorname{Pre}(\mathscr{C}) \rightleftarrows \Lambda \operatorname{Pre}(\mathscr{C}): \Phi_{r}^{\mathscr{C}}
$$


To wit, $\mathrm{sd}_{r}: \Delta^{\mathrm{op}} \rightarrow \Delta^{\mathrm{op}}, i: \Delta^{\mathrm{op}} \rightarrow \Lambda^{\mathrm{op}}$ and $C_{r} \rightarrow 1$ (the trivial group) induce $\left(C_{r} \rightarrow 1\right)_{\mathscr{C}}^{*}: \Delta \operatorname{Pre}(\mathscr{C}) \rightarrow\left[C_{r}, \Delta \operatorname{Pre}(\mathscr{C})\right]$ and $\left(i \circ \operatorname{sd}_{r}\right)_{\mathscr{C}}^{*}: \Lambda \operatorname{Pre}(\mathscr{C}) \rightarrow$ $\left[C_{r}, \Delta \operatorname{Pre}(\mathscr{C})\right]$. Taking the right Kan extension of $\left(C_{r} \rightarrow 1\right)_{\mathscr{C}}^{*}$ and the left Kan extension of $\left(i \circ \mathrm{sd}_{r}\right)_{\mathscr{C}}^{*}$ we get adjoint functor pairs:

$$
\Psi_{r}^{\mathscr{C}}: \Delta \operatorname{Pre}(\mathscr{C}) \underset{\left(C_{r} \rightarrow 1\right)_{*}^{\mathscr{C}}}{\stackrel{\left(C_{r} \rightarrow 1\right)_{\mathscr{C}}^{*}}{\rightleftarrows}}\left[C_{r}, \Delta \operatorname{Pre}(\mathscr{C})\right] \underset{\left(i \circ \mathrm{sd}_{r}\right)_{\mathscr{C}}^{*}}{\stackrel{\left(i o d_{r}\right)_{!}^{\mathscr{C}}}{\rightleftarrows}} \Lambda \operatorname{Pre}(\mathscr{C}): \Phi_{r}^{\mathscr{C}}
$$

Here $\left[C_{r}, \Delta \operatorname{Pre}(\mathscr{C})\right]$ denotes the functor category from the category with one object and morphisms the cyclic group of order $r$ to simplicial presheaves on $\mathscr{C}$. Note that the right adjoint $\left(C_{r} \rightarrow 1\right)_{*}^{\mathscr{C}}$ is the $C_{r}$-fixed point functor. Clearly (3) specializes to (2) when $\mathscr{C}$ is the one-point site. The right adjoint $\Phi_{1}^{\mathscr{C}}$ is the forgetful functor from cyclic to simplicial presheaves on $\mathscr{C}$. Using the special case of the one-point site, one checks easily that the left adjoint $\Psi_{r}^{\mathscr{C}}$ preserves monomorphisms for all $r \geq 1$.

\section{Pointwise model structures}

In this section we discuss the injective and projective pointwise model structures on $\Lambda \operatorname{Pre}(\mathscr{C})$. The pointwise model structures do not reflect the inner workings of Grothendieck sites, but they are pivotal for the construction of the finer local model structures in Section 5. Throughout the following, a model structure on cyclic sets refers to any of the four types of model structures in Section 2.

Definition 4.1. A map $\mathscr{X} \rightarrow \mathscr{Y}$ in $\Lambda \operatorname{Pre}(C)$ is a pointwise weak equivalence if the induced map $\mathscr{X}(C) \rightarrow \mathscr{Y}(C)$ is a weak equivalence between cyclic sets for every object $C \in \mathscr{C}$. The classes of pointwise cofibrations and fibrations are defined similarly.

The next result can be verified in analogy with [2, Theorems 1.17, 1.19].

THEOREM 4.2. The following classes of maps between cyclic presheaves define combinatorial and proper model structures on $\Lambda$ Pre $(\mathscr{C})$.

(i) Pointwise weak equivalences and pointwise cofibrations.

(ii) Pointwise weak equivalences and pointwise fibrations.

REMARK 4.3. The model structure in (i) is called the pointwise injective model structure. We note the injective fibrations are defined by the right lifting property with respect to pointwise acyclic cofibrations. In the pointwise projective model structure (ii), the projective cofibrations are defined by the left lifting property with respect to pointwise acyclic fibrations.

The following lemmas are now immediate from the definitions. 
LEMMA 4.4. With the weak level $r$ and weak monic level $r$ model structure on cyclic sets, the adjunction (3) is a Quillen pair for the pointwise injective and projective model structures.

LEMMA 4.5. The identity functor on $\Lambda$ Pre $(\mathscr{C})$ induces a Quillen equivalence between the pointwise injective and projective model structures.

Lemma 2.2 implies immediately:

LEMma 4.6. With a monic model structure on $\Lambda$ Set the pointwise injective model structure on $\Lambda$ Pre $(\mathscr{C})$ is monoidal.

Next we exhibit a well known set of generators for the pointwise projective model structure on $\Lambda$ Pre $(\mathscr{C})$.

LEMMA 4.7. In the pointwise projective model structure on $\Lambda \operatorname{Pre}(\mathscr{C})$ the class of cofibrations is generated by the set of maps $C \otimes i$ where $i$ is a generating cofibration in $\Lambda$ Set and $C \in \mathscr{C}$, while the class of acyclic cofibrations is generated by the set of maps $C \otimes j$ where $j$ is a generating acyclic cofibration in $\Lambda$ Set and $C \in \mathscr{C}$.

Using Lemma 4.7, we get:

LEMMA 4.8. With a monic model structure on $\Lambda$ Set the pointwise projective model structure on $\Lambda$ Pre $(\mathscr{C})$ is monoidal.

Standard adjunction arguments involving $\underline{\operatorname{Hom}}_{\Lambda \mathbf{P r e}(\mathscr{C})}$ and hom $\mathbf{h S e t}_{\text {Sem- }}$ bined with Lemma 4.6 and Lemma 4.8 imply:

COROLlary 4.9. With a monic model structure on $\Lambda$ Set the following equivalent statements hold true.

(i) If $i: \mathscr{X} \rightarrow \mathscr{Y}$ a cofibration and $i^{\prime}: \mathscr{X}^{\prime} \rightarrow \mathscr{Y}^{\prime}$ a fibration in either one of the pointwise model structures on $\Lambda$ Pre $(\mathscr{C})$, then the pullback map

$$
\begin{aligned}
& \underline{\operatorname{Hom}}_{\Lambda \operatorname{Pre}(\mathscr{C})}\left(\mathscr{Y}, \mathscr{X}^{\prime}\right) \\
& \quad \rightarrow \underline{\operatorname{Hom}}_{\Lambda \operatorname{Pre}(\mathscr{C})}\left(\mathscr{Y}, \mathscr{Y}^{\prime}\right) \times_{\underline{\operatorname{Hom}}_{\Lambda \operatorname{Pre}(\mathscr{C})}\left(\mathscr{X}, \mathscr{Y}^{\prime}\right)} \underline{\operatorname{Hom}}_{\Lambda \operatorname{Pre}(\mathscr{C})}\left(\mathscr{X}, \mathscr{X}^{\prime}\right)
\end{aligned}
$$

is a fibration that is a pointwise weak equivalence if either $i$ or $i^{\prime}$ is so.

(ii) With the same assumptions as in (i), the pullback map

$$
\operatorname{hom}_{\Lambda \operatorname{Set}}\left(\mathscr{Y}, \mathscr{X}^{\prime}\right) \rightarrow \operatorname{hom}_{\Lambda \operatorname{Set}}\left(\mathscr{Y}, \mathscr{Y}^{\prime}\right) \times_{\operatorname{hom}_{\Lambda \mathrm{Set}}\left(\mathscr{X}, \mathscr{Y}^{\prime}\right)} \operatorname{hom}_{\Lambda \operatorname{Set}}\left(\mathscr{X}, \mathscr{X}^{\prime}\right)
$$

is a fibration that is a weak equivalence in the model structure on $\Lambda$ Set if either $i$ or $i^{\prime}$ is so.

(iii) If $i: K \rightarrow L$ a cofibration in $\Lambda$ Set and $i^{\prime}: \mathscr{X}^{\prime} \rightarrow \mathscr{Y}^{\prime}$ a fibration in $\Lambda \operatorname{Pre}(\mathscr{C})$, the map

$$
\mathscr{X}^{\prime L} \rightarrow \mathscr{X}^{\prime K} \times \mathscr{Y}^{\prime K} \mathscr{Y}^{\prime L}
$$


is a fibration that is a pointwise weak equivalence if either $i$ or $i^{\prime}$ is so. (iv) The pointwise model structures on $\Lambda \mathbf{P r e}(\mathscr{C})$ are cyclic model structures.

\section{Local model structures}

We shall construct the local model structures on cyclic presheaves as left Bousfield localizations of the pointwise injective and projective model structures. Existence of localized model structures on combinatorial and left proper model structures is known by work of Smith. We refer to [8] for homotopy localization techniques at large and to [2] for a concise introduction to Bousfield localization of (enriched) model categories; in particular, we will employ derived mapping object functors for the pointwise model structures in Section 4.

The next result due to Dugger-Isaksen [6, Proposition 7.2] uses Jardine's work on simplicial presheaves $[10, \S 1]$.

Proposition 5.1. A map $\mathscr{X} \rightarrow \mathscr{Y}$ between simplicial presheaves on $\mathscr{C}$ is a local acyclic fibration if and only if every diagram

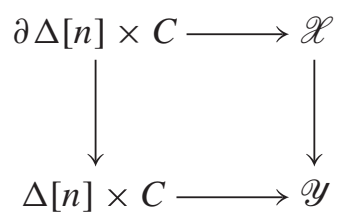

admits local liftings, i.e., there is a covering sieve $R \subset \mathscr{C}(-, C)$ such that for every $C^{\prime} \rightarrow C$ in $R$ there exists a lifting as indicated in the following diagram:

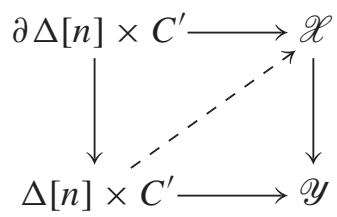

From [5, Definition 4.2] we recall:

Definition 5.2. A map of simplicial presheaves $\mathscr{U} \rightarrow C$ is a hypercover if it is a local acyclic fibration, $\mathscr{U}_{n}$ a coproduct of representables for every $n \geq 0$, and $C$ an object of $\mathscr{C}$.

In what follows we shall employ the classical Čech construction in order to define level descent conditions for cyclic presheaves. First we need some terminology: By a "pointwise level $r$ " model structure on $\Lambda$ Pre $(\mathscr{C})$ we refer to the pointwise injective and projective model structures in Theorem 4.2 in 
the event $\Lambda$ Set is equipped with either the "weak level $r$ " or the "weak monic level $r$ " model structure.

Definition 5.3. A pointwise level $r$ fibrant cyclic presheaf $\mathscr{Z}$ satisfies level $r$ descent for a hypercover $\mathscr{U} \rightarrow C$ if the functorially induced map of cyclic sets from $\mathscr{Z}(C)$ to the homotopy limit of the Čech construction

$$
\mathscr{Z}(C) \longrightarrow \operatorname{holim}\left(\prod_{\alpha_{0}} \mathscr{Z}\left(\mathcal{U}_{0}^{\alpha_{0}}\right) \rightleftarrows \prod_{\alpha_{1}} \mathscr{Z}\left(\mathcal{U}_{1}^{\alpha_{1}}\right) \rightleftarrows\right)
$$

is a level $r$ weak equivalence. The index $\alpha_{n}$ runs over the representable summands of $\mathscr{U}_{n}$. If $\mathscr{Z}$ is not pointwise level $r$ fibrant, then $\mathscr{Z}$ satisfies level $r$ descent if some pointwise level $r$ fibrant replacement satisfies level $r$ descent. A cyclic presheaf satisfies level $r$ descent if it satisfies level $r$ descent for every hypercover.

REMARK 5.4. If $\mathscr{Z}$ is a pointwise level $r$ fibrant cyclic presheaf, then $\Phi_{r}^{\mathscr{C}} \mathscr{Z}$ is a pointwise fibrant simplicial presheaf according to the Quillen adjunction (3) in Lemma 4.4. Thus $\mathscr{Z}$ satisfies level $r$ descent only if the simplicial presheaf $\Phi_{r}^{\mathscr{C}} \mathscr{Z}$ satisfies descent in the sense of [5, Definition 4.3].

What follows is a crux input in our approach to the local model structures on $\Lambda$ Pre $(\mathscr{C})$. It is the cyclic version of an analogous result for simplicial presheaves proven in [5, Lemma 4.1], which we refer to for further details.

LEMMA 5.5. A cyclic presheaf $\mathscr{Z}$ on $\mathscr{C}$ satisfies level $r$ descent for a hypercover $\mathscr{U} \rightarrow C$ if and only if the functorially induced map between derived mapping object functors

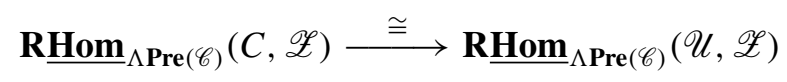

is an isomorphism for the pointwise level $r$ model structure on $\Lambda$ Pre $(\mathscr{C})$. In particular, $\mathscr{Z}$ satisfies level $r$ descent if and only if (5) is an isomorphism for every hypercover $\mathcal{U} \rightarrow C$.

Proof. In global sections the internal hom object $\underline{\operatorname{Hom}}_{\Lambda \mathbf{P r e}(\mathscr{C})}(C, \mathscr{Z})$ identifies with $\mathscr{Z}(C)$. Hence the lemma follows from the weak equivalences

$$
\begin{aligned}
\underline{\operatorname{Hom}}_{\Lambda \operatorname{Pre}(\mathscr{C})}(\mathscr{U}, \mathscr{Z}) & \simeq \underline{\operatorname{Hom}}_{\Lambda \operatorname{Pre}(\mathscr{C})}\left(\operatorname{hocolim} \mathscr{U}_{n}, \mathscr{Z}\right) \\
& \simeq \operatorname{holim} \underline{\operatorname{Hom}}_{\Lambda \operatorname{Pre}(\mathscr{C})}\left(\mathscr{U}_{n}, \mathscr{Z}\right) \\
& \simeq \operatorname{holim} \prod_{\alpha_{n}} \mathscr{Z}\left(\mathscr{U}_{n}^{\alpha_{n}}\right)
\end{aligned}
$$


Here, global sections and also cofibrant and fibrant replacements are left implicit in the notation.

Remark 5.6. There exist equivalent formulations of Lemma 5.5 in the pointwise injective and projective model structures in terms of $\operatorname{hom}_{\Lambda \mathrm{Set}}(-,-)$. We refer to [5, Lemma 4.1] for the corresponding formulations for simplicial presheaves.

We are ready to define level local weak equivalences for cyclic presheaves.

Definition 5.7. A map $\mathscr{X} \rightarrow \mathscr{Y}$ between cyclic presheaves on $\mathscr{C}$ is called a level $r$ local weak equivalence if for every cyclic presheaf $\mathscr{Z}$ that satisfies level $r$ descent, the functorially induced map between derived mapping objects

$$
\underline{\operatorname{RHom}}_{\Lambda \operatorname{Pre}(\mathscr{C})}(\mathscr{Y}, \mathscr{Z}) \longrightarrow \underline{\operatorname{Rom}}_{\Lambda \operatorname{Pre}(\mathscr{C})}(\mathscr{X}, \mathscr{Z})
$$

is an isomorphism for the pointwise level $r$ model structure on $\Lambda \operatorname{Pre}(\mathscr{C})$.

Remark 5.8. Every hypercover is a level $r$ local weak equivalence for every $r \geq 1$. The level $r$ local weak equivalences between cyclic presheaves that satisfies level $r$ descent are pointwise weak equivalences.

Next we shall define a countable infinite set worth of local model structures on $\Lambda \operatorname{Pre}(\mathscr{C})$ by localizing the pointwise model structures in Theorem 4.2 with respect to the class of hypercovers. The set-theoretic issues involved in these localizations can be dealt with by reference to [5]. In effect, the main body of work in [5] shows that for the second claim in Lemma 5.5 it suffices to consider only a dense set of hypercovers. The class of hypercovers has a dense subset by [5, Proposition 6.4]. We are ready to prove existence of the following model structures where the desired characterizations of the fibrant objects follow immediately from Lemma 5.5.

THEOREM 5.9. The following classes of maps define combinatorial and left proper Quillen equivalent model structures on $\Lambda$ Pre $(\mathscr{C})$.

(i) The level $r$ local injective model structure consisting of level $r$ local weak equivalences, pointwise cofibrations and level $r$ local injective fibrations. The class of level $r$ local injective fibrant cyclic presheaves coincides with the pointwise injective fibrant cyclic presheaves satisfying level $r$ descent.

(ii) The level $r$ local projective model structure consisting of level $r$ local weak equivalences, projective cofibrations and level $r$ local projective fibrations. The class of level $r$ local projective fibrant cyclic presheaves coincides with the pointwise fibrant cyclic presheaves satisfying level $r$ descent. 
Proof. The model structures are simply obtained by localizing the level $r$ model structures in Theorem 4.2 with respect to a dense subset of the class of hypercovers. Lemma 5.5 implies the classes of fibrant objects in the localized model structures coincide with the respective classes of level $r$ fibrant objects.

The constructions of the "strong" versions of the "level $r$ local" model structures on $\Lambda \operatorname{Pre}(\mathscr{C})$ run in parallel with the above: A "strong pointwise" model structure on cyclic presheaves refers to the pointwise injective and projective model structures in Theorem 4.2 in the event $\Lambda$ Set is equipped with either the "strong" or the "monic strong" model structure.

Definition 5.10. A strong pointwise fibrant cyclic presheaf $\mathscr{Z}$ satisfies strong descent for the hypercover $\mathscr{U} \rightarrow C$ if the canonical map in the Čech construction (4) is a strong pointwise weak equivalence. If $\mathscr{Z}$ is not strong pointwise fibrant, then $\mathscr{Z}$ satisfies strong descent if some strong pointwise fibrant replacement satisfies strong descent. A cyclic presheaf $\mathscr{Z}$ satisfies strong descent if it satisfies strong descent for every hypercover.

We leave the verification of the next result to the reader since it is analogous to the proof of Lemma 5.5.

Lemma 5.11. A cyclic presheaf $\mathscr{Z}$ on $\mathscr{C}$ satisfies strong descent for a hypercover $\mathscr{U} \rightarrow C$ if and only if the functorially induced map between derived mapping object functors

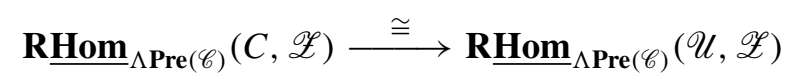

is an isomorphism for the strong pointwise model structure on $\Lambda \operatorname{Pre}(\mathscr{C})$.

Definition 5.12. A map $\mathscr{X} \rightarrow \mathscr{Y}$ between cyclic presheaves on $\mathscr{C}$ is called a strong local weak equivalence if for every cyclic presheaf $\mathscr{Z}$ that satisfies strong descent the functorially induced map between derived mapping object functors

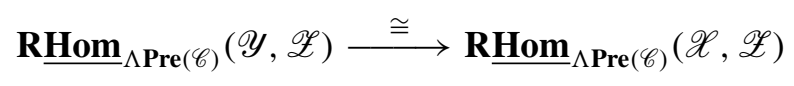

is an isomorphism for the strong pointwise model structure on $\Lambda \operatorname{Pre}(\mathscr{C})$.

We note that every hypercover is a strong local weak equivalence. With these definitions, a proof of the next result follows as for Theorem 5.9 by localizing the strong pointwise model structures with respect to a dense subset of the class of hypercovers on $\mathscr{C}$, and applying Lemma 5.11 for the identification of the strong local fibrant objects.

THEOREM 5.13. The following classes of maps define combinatorial and left proper Quillen equivalent model structures on $\Lambda$ Pre $(\mathscr{C})$. 
(i) The strong local injective model structure consisting of strong local weak equivalences, pointwise cofibrations and strong local injective fibrations (defined by the right lifting property). The class of strong local injective fibrant objects coincides with the strong pointwise injective fibrant cyclic presheaves satisfying strong descent.

(ii) The strong local projective model structure consisting of strong local weak equivalences, projective cofibrations and strong local projective fibrations (defined by the right lifting property). The class of strong local projective fibrant objects coincides with the strong pointwise projective fibrant cyclic presheaves satisfying strong descent.

We shall end this paper by noting that the local model structures are monoidal, and hence cyclic. This result suggests there exists a highly structured theory of stable homotopy of cyclic presheaves. For the one-point site, this should be closely related to stable $S^{1}$-equivariant homotopy theory.

Proposition 5.14. With a monic model structure on $\Lambda$ Set the local model structures on $\Lambda \operatorname{Pre}(\mathscr{C})$ are monoidal.

Proof. The proof of the corresponding result for simplicial presheaves given in [2, Theorem 3.38] carries over to the setting of cyclic presheaves on account of Lemma 4.6 and Lemma 4.8.

\section{REFERENCES}

1. Adámek, J., and Rosický, J., Locally Presentable and Accessible Categories, London Math. Soc. Lecture Note 189, Cambridge Univ. Press, Cambridge 1994.

2. Barwick, C., On (enriched) left Bousfield localization of model categories, arXiv:0708.2067.

3. Beke, T., Sheafifiable homotopy model categories, Math. Proc. Cambridge Philos. Soc. 129 (2000), 447-475.

4. Connes, A., Cohomologie cyclique et foncteurs $\mathrm{Ext}^{n}$, C. R. Acad. Sci. Paris (I) 296 (1983), 953-958.

5. Dugger, D., Hollander, S., and Isaksen, D. C., Hypercovers and simplicial presheaves, Math. Proc. Cambridge Philos. Soc. 136 (2004), 9-51.

6. Dugger, D., and Isaksen, D. C., Weak equivalences of simplicial presheaves, pp. 97-113 in: Homotopy Theory: Relations with Algebraic Geometry, Group Cohomology, and Algebraic $K$-theory (Proc. Evanston 2002), Contemp. Math. 346, Amer. Math. Soc., Providence, RI 2004.

7. Dwyer, W. G., Hopkins, M. J., and Kan, D. M., The homotopy theory of cyclic sets, Trans. Amer. Math. Soc. 291 (1985), 281-289.

8. Hirschhorn, P. S., Model Categories and their Localizations, Math. Surveys and Monographs 99, Amer. Math. Soc., Providence, RI 2003.

9. Hovey, M., Model Categories, Mathematical Surveys and Monographs 63, Amer. Math. Soc., Providence, RI 1999.

10. Jardine, J. F., Simplicial presheaves, J. Pure Appl. Algebra 47 (1987), 35-87. 
11. Jardine, J. F., Generalised sheaf cohomology theories, pp. 29-68 in: Axiomatic, Enriched and Motivic Homotopy Theory (Proc. Cambridge 2002), NATO Sci. Ser. II Math. Phys. Chem. 131, Kluwer, Dordrecht 2004.

12. Mac Lane, S., and Moerdijk, I., Sheaves in Geometry and Logic. A First Introduction to Topos Theory, Universitext, Springer, New York 1994.

13. Seteklev, S., Homotopy theories of cyclic objects, Master's thesis, University of Oslo, 2007.

14. Spaliński, J., Strong homotopy theory of cyclic sets, J. Pure Appl. Algebra 99 (1995), 35-52.

DEPARTMENT OF MATHEMATICS

UNIVERSITY OF OSLO

NORWAY

E-mail: sigurdts@student.matnat.uio.no

paularne@math.uio.no 